\title{
On approximation properties of a generalization of Bernstein polynomials in symmetric range
}

Ecem Acar and Aydin Izgi

Department of Mathematics, University of Harran, Sanliurfa, Turkey

Received: 28 December 2020, Accepted: 30 December 2020

Published online: 21 March 2021.

\begin{abstract}
In the present paper, in order to make the convergence faster to a function being approximated we identify a new generalization of Bernstein operators depending on symmetric range. The rate of convergence of these operators are given by using the modulus of continuity. Furthermore,we establish Korovkin-type approximation theorem and Voronovskaja type asymptotic theorem. Finally, we show that using graphics in Maple this new generalization of Bernstein operators converge faster than Bernstein operators on symmetric range for certain functions.
\end{abstract}

Keywords: Bernstein Operators, Modulus of continuity, Rate of convergence, Voronovskaja type theorem.

\section{Introduction}

In 1912, Bernstein [1] introduced the classical Bernstein polynomials $B_{n}(f ; x)$ for $f \in C[0,1]$ as below

$$
B_{n}(f ; x)=\sum_{k=0}^{n} f\left(\frac{k}{n}\right)\left(\begin{array}{l}
n \\
k
\end{array}\right) x^{k}(1-x)^{n-k}
$$

Bernstein polynomials can uniformly approximate any continuous function over a closed interval.

In the papers $[4,5,6]$, various generalization of Bernstein polynomials are investigated. Also, approximating continuous functions by classical Bernstein polynomials have been studied for two dimensional Bernstein polynomials in [7]-[9]. The idea of constructing linear and nonlinear positive operators have been studied intensively in approximation theory (see [11],[12]). A generalization of Bernstein polynomials in symmetric range are defined in [2]

$$
C_{n}(f ; x)=\sum_{k=0}^{n}\left(\begin{array}{l}
n \\
k
\end{array}\right)\left(\frac{1}{2}+\frac{x}{2}\right)^{k}\left(\frac{1}{2}-\frac{x}{2}\right)^{n-k} f\left(2 \frac{k}{n}-1\right)
$$

for $f \in C[-1,1]$ and $n \in \mathbb{N}$. These operators given in (2) are linear positive in symmetric range and provide the Korovkin theorem's conditions. Also, the operators (2) are smooth convergence on the range of $[-1,1]$.

The purpose of this paper is to introduce a new generalization of Bernstein polynomials in symmetric range and their certain elementary properties. One of our main important results is uniform convergence to a continuous function. Also, in that paper we calculate rate of convergence of this new generalization by using modulus of continuity and give the Voronovskaja type asymptotic theorem. 
Let $I=\left[-\frac{m+a}{m+b}, \frac{m+a}{m+b}\right], a, b \in \mathbb{N}$ and $f \in C[I]$. Then we define the linear positive operators $E_{m}(f ; x)$ in the following way

$$
E_{m}(f ; x)=\frac{1}{2^{m}}\left(\frac{m+b}{m+a}\right)^{m} \sum_{j=0}^{m} p_{m, j, a, b}(x) f\left(\left(2 \frac{j}{m}-1\right) \frac{m+a}{m+b}\right)
$$

which

$$
p_{m, j, a, b}(x)=\left(\begin{array}{c}
m \\
j
\end{array}\right)\left(x+\frac{m+a}{m+b}\right)^{j}\left(\frac{m+a}{m+b}-x\right)^{m-j}, j=0,1, \ldots, m .
$$

It is clear that let take $a=b=0$ for the operators given (3) then it reduce to Bernstein polynomials in symmetric range given in [2].

In the following section, we obtain the properties of approximation of these operators.

\section{Main results}

Lemma 1. For $\forall x \in I$ and $m \in \mathbb{N}$, symmetric Bernstein operators (3) is satisfied the following equalities:

$$
E_{m}(1 ; x)=1, \quad E_{m}(t ; x)=x
$$

and

$$
E_{m}\left(t^{2} ; x\right)=x^{2}+\frac{\left(\frac{m+a}{m+b}\right)^{2}-x^{2}}{m}
$$

Proof. It is clear that $E_{m}(1 ; x)=1$.

$$
\begin{aligned}
E_{m}(t ; x) & =\frac{1}{2^{m}}\left(\frac{m+b}{m+a}\right)^{m} \sum_{j=0}^{m} p_{m, j, a, b}(x)\left(\left(2 \frac{j}{m}-1\right) \frac{m+a}{m+b}\right) \\
& =\frac{1}{2^{m-1}}\left(\frac{m+b}{m+a}\right)^{m-1} \sum_{j=0}^{m} p_{m, j, a, b}(x) \frac{j}{m}-\frac{1}{2^{m}}\left(\frac{m+b}{m+a}\right)^{m-1} \sum_{j=0}^{m} p_{m, j, a, b}(x) \\
& =\frac{1}{2^{m-1}}\left(\frac{m+b}{m+a}\right)^{m-1}\left(x+\frac{m+a}{m+b}\right) \sum_{j=0}^{m-1}\left(\begin{array}{c}
m-1 \\
j
\end{array}\right)\left(x+\frac{m+a}{m+b}\right)^{j} \times\left(\frac{m+a}{m+b}-x\right)^{m-j}-\frac{m+a}{m+b} \\
& =x \\
E_{m}\left(t^{2} ; x\right) & =\frac{1}{2^{m}}\left(\frac{m+b}{m+a}\right)^{m} \sum_{j=0}^{m} p_{m, j, a, b}(x)\left(4 \frac{j^{2}}{m^{2}}-4 \frac{j}{m}+1\right) \frac{(m+a)^{2}}{(m+b)^{2}} \\
& =\frac{1}{2^{m-2}}\left(\frac{m+b}{m+a}\right)^{m-2} \sum_{j=0}^{m} p_{m, j, a, b}(x)\left(\frac{j(j-1)}{m^{2}}+\frac{j}{m^{2}}\right)^{m} \\
& -\frac{1}{2^{m-2}}\left(\frac{m+b}{m+a}\right)^{m-2} \sum_{j=0}^{m} p_{m, j, a, b}(x) \frac{j}{m}+\frac{1}{2^{m}}\left(\frac{m+b}{m+a}\right)^{m-2} \sum_{j=0}^{m} p_{m, j, a, b}(x) \\
& =\frac{1}{2^{m-2}}\left(\frac{m+b}{m+a}\right)^{m-2} \sum_{j=2}^{m} \frac{m \cdot(m-1) !}{(m-j) ! j(j-1)(j-2) !}\left(x+\frac{m+a}{m+b}\right)^{j} \times\left(\frac{m+a}{m+b}-x\right)^{m-j} \frac{j(j-1)}{m^{2}}
\end{aligned}
$$




$$
\begin{aligned}
& +\frac{1}{2^{m-2}}\left(\frac{m+b}{m+a}\right)^{m-2} \sum_{j=1}^{m} \frac{m \cdot(m-1) !}{(m-j) ! j(j-1) !}\left(x+\frac{m+a}{m+b}\right)^{j} \times\left(\frac{m+a}{m+b}-x\right)^{m-j} \frac{j}{m^{2}} \\
& -\frac{1}{2^{m-2}}\left(\frac{m+b}{m+a}\right)^{m-2} \sum_{j=1}^{m} \frac{m \cdot(m-1) !}{(m-j) ! j(j-1) !}\left(x+\frac{m+a}{m+b}\right)^{j} \times\left(\frac{m+a}{m+b}-x\right)^{m-j} \frac{j}{m}+\left(\frac{m+a}{m+b}\right)^{2} \\
= & \frac{1}{2^{m-2}}\left(\frac{m+b}{m+a}\right)^{m-2} \frac{m-1}{m} \sum_{j=0}^{m-2}\left(\begin{array}{c}
m-2 \\
j
\end{array}\right)\left(x+\frac{m+a}{m+b}\right)^{j+2}\left(\frac{m+a}{m+b}-x\right)^{m-j-2} \\
& +\frac{1}{2^{m-2}}\left(\frac{m+b}{m+a}\right)^{m-2} \frac{1}{m} \sum_{j=0}^{m-1}\left(\begin{array}{c}
m-1 \\
j
\end{array}\right)\left(x+\frac{m+a}{m+b}\right)^{j+1}\left(\frac{m+a}{m+b}-x\right)^{m-j-1} \\
& -\frac{1}{2^{m-2}}\left(\frac{m+b}{m+a}\right)^{m-2} \sum_{j=0}^{m-1}\left(\begin{array}{c}
m-1 \\
j
\end{array}\right)\left(x+\frac{m+a}{m+b}\right)^{j+1}\left(\frac{m+a}{m+b}-x\right)^{m-j-1}+\left(\frac{m+a}{m+b}\right)^{2} \\
= & \frac{m-1}{m} \frac{1}{2^{m-2}}\left(\frac{m+b}{m+a}\right)^{m-2}\left(x+\frac{m+a}{m+b}\right)^{2} \sum_{j=0}^{m-2}\left(\begin{array}{c}
m-2 \\
j
\end{array}\right) \times\left(x+\frac{m+a}{m+b}\right)^{j}\left(\frac{m+a}{m+b}-x\right)^{m-j-2} \\
& +\frac{1}{m} \frac{1}{2^{m-2}}\left(\frac{m+b}{m+a}\right)^{m-2}\left(x+\frac{m+a}{m+b}\right)^{m-1} \sum_{j=0}^{m-1}\left(\begin{array}{c}
m-1 \\
j
\end{array}\right) \times\left(x+\frac{m+a}{m+b}\right)^{j}\left(\frac{m+a}{m+b}-x\right)^{m-j-1} \\
& -\frac{1}{2^{m-2}}\left(\frac{m+b}{m+a}\right)^{m-2}\left(x+\frac{m+a}{m+b}\right)_{j=0}^{m-1}\left(\begin{array}{c}
m-1 \\
j
\end{array}\right) \times\left(x+\frac{m+a}{m+b}\right)^{j}\left(\frac{m+a}{m+b}-x\right)^{m-j-1}+\left(\frac{m+a}{m+b}\right)^{2} \\
= & \left(1-\frac{1}{m}\right)\left(x^{2}-\left(\frac{m+a}{m+b}\right)^{2}\right)+\left(\frac{m+a}{m+b}\right)^{2}=x^{2}+\frac{\left(\frac{m+a}{m+b}\right)^{2}-x^{2}}{m}
\end{aligned}
$$

Theorem 1. For $f \in C(I)$, the operators $E_{m}(f ; x)$ converges uniformly to $f(x)$ in respect to $x \in I$.

Proof. From the Lemma 1, we have

$$
\lim _{m \rightarrow \infty} \max _{x \in I}\left|E_{m}\left(t^{2} ; x\right)-x^{2}\right|=\lim _{m \rightarrow \infty} \max _{x \in I}\left|\frac{\left(\frac{m+a}{m+b}\right)^{2}-x^{2}}{m}\right|=0
$$

Hence, we get

$$
\lim _{m \rightarrow \infty} \max _{x \in I}\left|E_{m}\left(t^{v} ; x\right)-x^{v}\right|=0, \quad v=0,1,2 .
$$

Accordingly the Bohman-Korovkin theorem [3], we get the desired result.

Lemma 2. Let obtain the following results for the central moments of the $E_{m}(f ; x)$ operators defined by

$$
C_{m, p}(x)=E_{m}\left((t-x)^{p} ; x\right), \quad p=0,1,2 .
$$

Then we get

$$
\begin{gathered}
C_{m, 0}(x)=1, C_{m, 1}(x)=0, \\
C_{m, 2}(x)=\frac{(m+a)^{2}-x^{2}(m+b)^{2}}{m(m+b)^{2}} .
\end{gathered}
$$

Also, the equality

$$
\lim _{m \rightarrow \infty} E_{m}\left((t-x)^{2} ; x\right)=\left(\frac{m+a}{m+b}\right)^{2}-x^{2}
$$

is satisfied.

Proof. It is obvious that

$$
C_{m, 0}(x)=1, C_{m, 1}(x)=0,
$$


hold from Lemma 1. From (6), we obtain

$$
\begin{aligned}
C_{m, 2}(x)= & E_{m}\left((t-x)^{2} ; x\right)=\frac{1}{2^{m}}\left(\frac{m+b}{m+a}\right)^{m} \sum_{j=0}^{m} p_{m, j, a, b}(x)\left(\left(2 \frac{j}{m}-1\right) \frac{m+a}{m+b}-x^{2}\right) \\
= & \frac{1}{2^{m}}\left(\frac{m+b}{m+a}\right)^{m} \sum_{j=0}^{m} p_{m, j, a, b}(x)\left(\left(2 \frac{j}{m}-1\right) \frac{m+a}{m+b}\right)-\frac{x}{2^{m-1}}\left(\frac{m+b}{m+a}\right)^{m} \sum_{j=0}^{m} p_{m, j, a, b}(x)\left(\left(2 \frac{j}{m}-1\right) \frac{m+a}{m+b}\right) \\
& +\frac{x^{2}}{2^{m}}\left(\frac{m+b}{m+a}\right)^{m} \sum_{j=0}^{m} p_{m, j, a, b}(x)=\frac{(m+a)^{2}-(m+b)^{2} x^{2}}{(m+b)^{2} m}
\end{aligned}
$$

Furthermore, we get

$$
\lim _{m \rightarrow \infty} m E_{m}\left((t-x)^{2} ; x\right)=\lim _{m \rightarrow \infty} \frac{m\left((m+a)^{2}-(m+b)^{2} x^{2}\right)}{m(m+b)^{2}}=\left(\frac{m+a}{m+b}\right)^{2}-x^{2}
$$

Theorem 2. If $f \in C(I)$, then the below inequality holds

$$
\left|E_{m}(f ; x)-f(x)\right| \leq\left(1+\frac{m+a}{m+b}\right) \omega\left(f ; \frac{1}{\sqrt{m}}\right) .
$$

Proof. Let $f \in C(I)$, we get

$$
\left|E_{m}(f ; x)-f(x)\right|=\left|\frac{1}{2^{m}}\left(\frac{m+b}{m+a}\right)^{m} \sum_{j=0}^{m} p_{m, j, a, b}(x) f\left(\left(2 \frac{j}{m}-1\right) \frac{m+a}{m+b}\right)-f(x) \frac{1}{2^{m}}\left(\frac{m+b}{m+a}\right)^{m} \sum_{j=0}^{m} p_{m, j, a, b}(x)\right| .
$$

Since $\frac{1}{2^{m}} \geq 0$ and $p_{m, j, a, b}(x) \geq 0$ and using e Cauchy-Schwartz inequality, we obtain

$$
\left|E_{m}(f ; x)-f(x)\right|=\frac{1}{2^{m}}\left(\frac{m+b}{m+a}\right)^{m} \sum_{j=0}^{m}\left|f\left(\left(2 \frac{j}{m}-1\right) \frac{m+a}{m+b}\right)-f(x)\right| p_{m, j, a, b}(x) .
$$

From the properties of modulus of continuity, we can write

$$
\left|f\left(\left(2 \frac{j}{m}-1\right) \frac{m+a}{m+b}\right)-f(x)\right| \leq\left(\frac{\left|\left(2 \frac{j}{m}-1\right) \frac{m+a}{m+b}-x\right|}{\delta}+1\right) \omega(f ; \delta)
$$

Now, replacement in the inequality (7), we obtain

$$
\begin{aligned}
\left|E_{m}(f ; x)-f(x)\right| \leq & \frac{1}{2^{m}}\left(\frac{m+b}{m+a}\right)^{m} \sum_{j=0}^{m}\left(\frac{\left|\left(2 \frac{j}{m}-1\right) \frac{m+a}{m+b}-x\right|}{\delta}+1\right) \omega(f ; \delta) p_{m, j, a, b}(x) \\
= & \frac{1}{2^{m}}\left(\frac{m+b}{m+a}\right)^{m} \sum_{j=0}^{m} \frac{\left|\left(2 \frac{j}{m}-1\right) \frac{m+a}{m+b}-x\right|}{\delta} \omega(f ; \delta) p_{m, j, a, b}(x) \\
& +\frac{1}{2^{m}}\left(\frac{m+b}{m+a}\right)^{m} \sum_{j=0}^{m} \omega(f ; \delta) p_{m, j, a, b}(x) \\
= & \omega(f ; \delta)\left(\frac{1}{\delta 2^{m}}\left(\frac{m+b}{m+a}\right)^{m} \sum_{j=0}^{m}\left|\left(2 \frac{j}{m}-1\right) \frac{m+a}{m+b}-x\right| p_{m, j, a, b}(x)+1\right)
\end{aligned}
$$


Let take

$$
\begin{aligned}
A & =\frac{1}{2^{m}}\left(\frac{m+b}{m+a}\right)^{m} \sum_{j=0}^{m}\left|\left(2 \frac{j}{m}-1\right) \frac{m+a}{m+b}-x\right| p_{m, j, a, b}(x) \\
& =\sum_{j=0}^{m}\left(\left|\left(2 \frac{j}{m}-1\right) \frac{m+a}{m+b}-x\right|^{2}\right)^{1 / 2}\left(\frac{1}{2^{m}}\left(\frac{m+b}{m+a}\right)^{m} p_{m, j, a, b}(x)\right)^{1 / 2}\left(\frac{1}{2^{m}}\left(\frac{m+a}{m+b}\right)^{m} p_{m, j, a, b}(x)\right)^{1 / 2} .
\end{aligned}
$$

Applying Cauchy-Schwarz inequality, we get

$$
\begin{aligned}
A & \leq\left(\sum_{j=0}^{m}\left|\left(2 \frac{j}{m}-1\right) \frac{m+a}{m+b}-x\right|^{2}\left(\frac{1}{2^{m}}\left(\frac{m+a}{m+b}\right)^{m} p_{m, j, a, b}(x)\right)\right)^{1 / 2} \times\left(\sum_{j=0}^{m} \frac{1}{2^{m}}\left(\frac{m+a}{m+b}\right)^{m} p_{m, j, a, b}(x)\right)^{1 / 2} \\
& =\left(\frac{1}{2^{m}}\left(\frac{m+a}{m+b}\right)^{m} \sum_{j=0}^{m}\left|\left(2 \frac{j}{m}-1\right) \frac{m+a}{m+b}-x\right|^{2} p_{m, j, a, b}(x)\right)^{1 / 2} \\
& =\left(\frac{1}{2^{m}}\left(\frac{m+a}{m+b}\right)^{m} \sum_{j=0}^{m}\left(\left(\left(2 \frac{j}{m}-1\right) \frac{m+a}{m+b}\right)^{2}-2 x\left(2 \frac{j}{m}-1\right) \frac{m+a}{m+b}+x^{2}\right) p_{m, j, a, b}(x)\right)^{1 / 2} \\
& =\left(E_{m}\left(t^{2} ; x\right)-2 x E_{m}(t ; x)+x^{2} E_{m}(1 ; x)\right)^{1 / 2} \\
& =\left(\frac{\left(\frac{m+a}{m+b}\right)^{2}-x^{2}}{m}\right)^{1 / 2} .
\end{aligned}
$$

Therefore, we get

$$
\left|E_{m}(f ; x)-f(x)\right| \leq \omega(f ; \delta)\left(\frac{1}{\delta}\left(\frac{\left(\frac{m+a}{m+b}\right)^{2}-x^{2}}{m}\right)^{1 / 2}+1\right) .
$$

Also, we obtain $\max \left\{\left(\left(\frac{m+a}{m+b}\right)^{2}-x^{2}\right)^{1 / 2}\right\}=\frac{m+a}{m+b}$ for $x \in[I]$. Hence, choosing $\delta=\frac{1}{\sqrt{m}}$, we get the desired result as below

$$
\left|E_{m}(f ; x)-f(x)\right| \leq \omega(f ; \delta)\left(1+\frac{m+a}{m+b}\right) .
$$

Theorem 3. Voronovskaja Type Theorem. For $f \in C^{2}(I)$, we obtain the following equality

$$
\lim _{m \rightarrow \infty} m .\left(E_{m}(f ; x)-f(x)\right)=\frac{1}{2}\left(\left(\frac{m+a}{m+b}\right)^{2}-x^{2}\right) f^{\prime \prime}(x) .
$$

Proof. Taylor expansion of a function $f$ at point $x$ is as follows

$$
f(t)=f(x)+f^{\prime}(x)(t-x)+\frac{1}{2} f^{\prime \prime}(x)(t-x)^{2}+r(t ; x)(t-x)^{2}
$$

where $r(t ; x)$ is the remainder term and it is clear that $\lim _{t \rightarrow x} r(t ; x)=0$ and $r(t ; x) \in C[I]$. By linearity of Bernstein operators (3) and taking $t=\left(2 \frac{j}{m}-1\right) \frac{m+a}{m+b}$, we get

$$
m .\left(E_{m}(f ; x)-f(x)\right)=m \cdot E_{m}((t-x) ; x) f^{\prime}(x)+m \cdot E_{m}\left((t-x)^{2} ; x\right) \frac{f^{\prime \prime}(x)}{2}+m \cdot E_{m}\left(r(t ; x)(t-x)^{2} ; x\right) .
$$


Applying Cauchy-Schwartz inequality to the last term on the right hand side, we have

$$
m \cdot E_{m}\left(r(t ; x)(t-x)^{2} ; x\right) \leq\left(m^{2} E_{m}\left((t-x)^{4} ; x\right)\right)^{1 / 2}\left(E_{m}\left(r(t ; x)^{2} ; x\right)\right)^{1 / 2}
$$

Since $r^{2}(x ; x)=0, r^{2}(. ; x) \in C[I]$ and from Theorem 1 , we can write

$$
\lim _{m \rightarrow \infty} E_{m}\left(r(t ; x)^{2} ; x\right)=r^{2}(x ; x)=0 .
$$

Using (8), (9) and Lemma 2, we get the following desired result

$$
\lim _{m \rightarrow \infty} m .\left(E_{m}(f ; x)-f(x)\right)=\frac{1}{2}\left(\left(\frac{m+a}{m+b}\right)^{2}-x^{2}\right) f^{\prime \prime}(x) .
$$

\section{Numerical examples}

In this section, using graphics in Maple some numerical examples are given. Therefore, we compare the convergence of the operators $E_{m}(f, a, b ; x)$ with $C_{n}(f ; x)$ defined in [2]. Based on this result we may say that the operators $E_{m}(f, a, b ; x)$ converge faster than $C_{n}(f ; x)$ operators for certain functions.

Example 1. Let $f(x)=e^{-x / 5} \sin (3 \pi x)$ for $m=75$ and $m=125$ the convergence of the operators $E_{m}(f ; x)$ is respectively shown in Fig 1 and Fig 2.

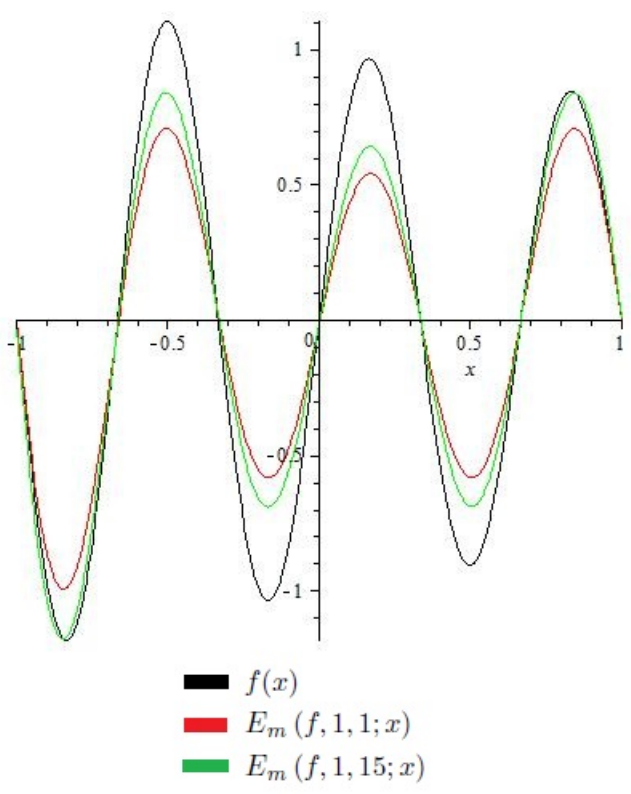

Fig. 1: The convergence of the $E_{m}(f ; x)$ operators for $f(x)=e^{-x / 5} \sin (3 \pi x)$ and $m=75$ 


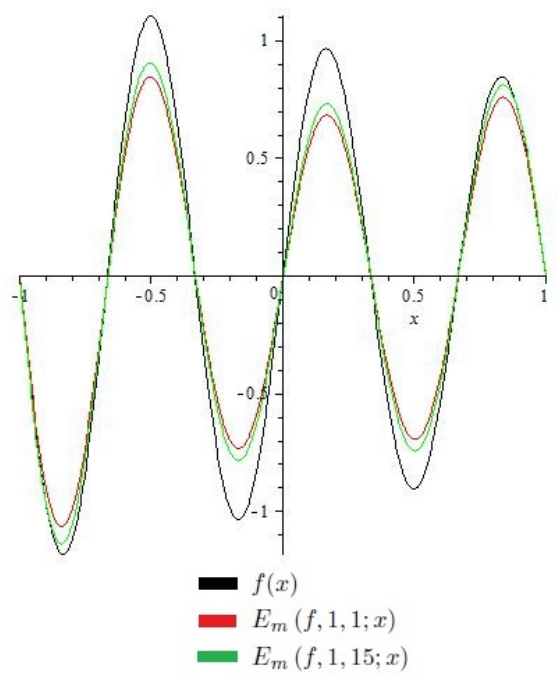

Fig. 2: The convergence of the $E_{m}(f ; x)$ operators for $f(x)=e^{-x / 5} \sin (3 \pi x)$ and $m=125$

\section{Competing interests}

The authors declare that they have no competing interests.

\section{Authors' contributions}

All authors have contributed to all parts of the article. All authors read and approved the final manuscript.

\section{References}

[1] Bernstein, S., Demonstration du theoreme de Weierstrass, fondeesur sur le calcul des probabilites, Commun. Soc. Math. Kharkov, $13(2), 1-2,1912$.

[2] Cilo, A., [-1,1] araliginda Bernstein polinomlarinin yaklasim ozellikleri ve yaklasim hizi, Master thesis, Harran University, (2012).

[3] Korovkin, P. P., On convergence of linear positive operators in the space of continuous functions. Dokl. Akad. Nauk 90:961-964 (1953).

[4] Cao, J. D. (1997). A generalization of the Bernstein Polynomials. J. Math. Anal. Appl. Math. 122, 1-21.

[5] Ilinskii, A. and Ostrovska, S., (2002). Convergence of generalized Bernstein polynomials. J. Approx. Theory 116, 100-112.

[6] Izgi,A. (2012). Approximation by a class of new type Bernstein polynomials of one two variables. Global J. Pure and Appl. Math. $8(5), 55-71$.

[7] Dirik, F. and Demirci, K., Korovkin type approximation theorem for functions of two variables in statistical sense, Turk. J. Math., 34 (2010), 73-83.

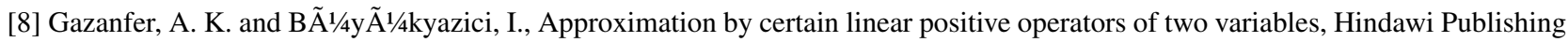
Corporation Abstract and Applied Analysis, ID 782080, (2014).

[9] Stancu, D. D., A method for obtaining polynomials of Bernstein type of two variables, Amer. Math. Monthly, 70(3) (1963), 260-264.

[10] Karahan, D. and Izgi, A., Approximation properties of Bernstein-Kantorovich type operators of two variables. Commun. Fac. Sci. Univ. Ank. Ser. A1. Math. Stat. 68 (2019), no. 2, 2313-2323.

[11] Aydin Ari, D. and Kirci Serenbay, S., Approximation by a generalization of the Jakimovski-Leviatan operators. Filomat 33(2019), no.8, 2345-2353.

[12] Acar, E., Karahan, D. and Kirci Serenbay, S., Approximation for the Bernstein operator of max-product kind in symmetric range. Khayyam Journal of Mathematics (2020) 6(2), 257-273. 